\title{
Efficacy and safety of statin therapy in pulmonary hypertension: a systematic review and meta-analysis
}

\author{
Fangying Chen ${ }^{1,2 \#}$, Mei Yang ${ }^{1 \#}$, Chun Wan ${ }^{1 \#}$, Lin Liu $^{3}$, Lei Chen ${ }^{1}$ \\ ${ }^{1}$ Department of Respiratory and Critical Care Medicine, West China Hospital, West China School of Medicine, Sichuan University, Chengdu \\ 610041, China; ${ }^{2}$ Department of Tuberculosis, the Third People's Hospital of Tibet Autonomous Region, Lhasa 850000, China; ${ }^{3}$ Department of \\ Respiratory and Critical Care Medicine, 363 Hospital, Chengdu 610041, China \\ Contributions: (I) Conception and design: L Liu, L Chen; (II) Administrative support: None; (III) Provision of study materials or patients: None; \\ (IV) Collection and assembly of data: F Chen, M Yang; (V) Data analysis and interpretation: F Chen, M Yang, C Wan; (VI) Manuscript writing: All \\ authors; (VII) Final approval of manuscript: All authors. \\ \#These authors contributed equally to this work. \\ Correspondence to: Lei Chen. Department of Respiratory and Critical Care Medicine, West China Hospital, West China School of Medicine, Sichuan \\ University, Chengdu 610041, China. Email: lchens@126.com; Lin Liu. Department of Respiratory and Critical Care Medicine, 363 Hospital, \\ Chengdu 610041, China. Email: 1liniu@126.com.
}

Background: Pulmonary hypertension $(\mathrm{PH})$ is a multi-causal disease and no satisfactory therapeutic strategies for it. Statins have been suggested as potential drugs in $\mathrm{PH}$, whose effects in different clinic types of $\mathrm{PH}$ have not been conclusive. In this study, we included randomized controlled clinical trials (RCTs) evaluating the efficacy and safety of statins therapy in $\mathrm{PH}$.

Methods: We searched databases including Medline, Embase, Cochrane, PubMed and Web of science, with time up to January 1, 2019. With 95\% confidence interval (CI), weighted mean difference (WMD) or standardized mean difference (SMD) was pooled and calculated in a random or fixed effect model according to I2 statistic.

Results: A total of nine RCTs with 657 patients were included. Four types of statins (atorvastatin, pravastatin, rosuvastatin and simvastatin) were used at different doses (10-80 $\mathrm{mg}$ daily) for up to 6 months. In the pooled-data analysis, compared with placebo, there were significant improvements in pulmonary arterial pressure (PAP), in addition to low-density lipoprotein (LDL) in patients treated with statins, but not in 6-minute walking distance (6MWD), cardiac index (CDI). No more adverse events and all-cause mortality were revealed. Subgroup analysis indicated that statins could decrease PAP in the subtype of PH due to chronic obstructive pulmonary disease (COPD), but not pulmonary arterial hypertension (PAH).

Conclusions: This study indicates that statins can efficiently and safely reduce PAP in PH, especially in the subtype due to COPD. Further RCTs are needed to focus on the efficacy and safety of statin therapy in different subtypes of $\mathrm{PH}$.

Keywords: Pulmonary hypertension (PH); chronic obstructive pulmonary disease (COPD); pulmonary arterial hypertension $(\mathrm{PAH})$; statins; meta-analysis

Submitted Jul 03, 2019. Accepted for publication Oct 17, 2019.

doi: $10.21037 /$ atm.2019.11.19

View this article at: http://dx.doi.org/10.21037/atm.2019.11.19

\section{Introduction}

Pulmonary hypertension $(\mathrm{PH})$ is a fatal disorder characterized by persistently increased pulmonary arterial pressure (PAP) of various causes, which is defined as an increase of the mean PAP above $25 \mathrm{mmHg}$ at rest (1). According to primary causes, $\mathrm{PH}$ is categorized into five clinic subtypes (2): (I) pulmonary arterial hypertension (PAH), including idiopathic, heritable forms and $\mathrm{PAH}$ 
associated with connective tissue disease and congenital heart disease, etc.; (II) $\mathrm{PH}$ due to left heat disease; (III) $\mathrm{PH}$ due to lung diseases and/or hypoxia [chronic obstructive pulmonary disease (COPD), etc.]; (IV) chronic thromboembolic $\mathrm{PH}(\mathrm{CTPH})$ and other pulmonary artery obstructions; (V) PH with unclear and/or multifactorial mechanisms. These subtypes give rise to mechanisms regarding vasoconstriction, vascular wall thickening, stenosis, along with remodeling, thrombosis and blood viscosity and volume increasing caused by chronic hypoxia. The long-term progression would produce aggravated cardiac load, ultimately, cor pulmonale which severely affects the respiratory and cardiovascular systems. Current routine medications for $\mathrm{PH}$ include vasodilators, such as endothelin receptor antagonists, prostacyclin analogues (prostanoids) and their likes $(3,4)$, anticoagulants, diuretics, cardiotonic agents and oxygen, etc. However, the prognosis has been always unsatisfactory since the lack of mechanismtargeted therapy. Besides, given the treatments of $\mathrm{PH}$ differing with respect to their types, more effective and targeted therapy is urgently needed.

Statins, as HMG-CoA reductase inhibitors, have pleiotropic effects, such as lipid-lowering, anti-proliferative, antioxidant, anti-inflammatory and endothelial cell functions maintaining properties (5-7), which may be helpful in attenuating the progression of $\mathrm{PH}$. It was reported that statins can prevent and reverse pulmonary vascular remodeling in several animal models of $\mathrm{PH}$ (8). An observational study showed there were significant improvements in 6-minute walking distance (6MWD), cardiac output and right ventricular systolic pressures in $\mathrm{PH}$ treated with simvastatin (9). However, Anand et al. found that there was no significant improvement in 6MWD and mortality with statin therapy, compared to placebo (10). Some meta-analyses showed that statins treatment had no effect of PAP in PH patients (11-13). An increasing number of studies have been focused on statin therapy in $\mathrm{PH}$ these years, even though the results were controversial (14-16). And statins have been suggested as novel and effective drugs for $\mathrm{PH}$ in some vivo and vitro studies (5-7).

Given the undetermined situation, we performed this systematic review and meta-analysis to evaluate the efficacy and safety of statin therapy in randomized controlled trials (RCTs) of $\mathrm{PH}$ and a subgroup analysis concerning $\mathrm{PAH}$ and $\mathrm{PH}$ due to COPD, with the expectation to provide evidence regarding the role of statin therapy in $\mathrm{PH}$, especially those due to PAH and COPD.

\section{Methods}

\section{Search strategies}

We searched Medline (1946 to December week 4, 2018), Embase (1974 to January 2019), Cochrane controlled trials register (The Cochrane Library Issue 1, 2019), and PubMed (updated to January, 2019), Web of science (1990-2019) for eligible articles, using the terms: "pulmonary hypertension", "pulmonary arterial hypertension", and "PAH", combined with the following individual search terms: "HMG-CoA reductase inhibitor", "statin", "statins"; "atorvastatin", "cerivastatin", "fluvastatin", "lovastatin", "pravastatin", "pitavastatin", "rosuvastatin", "simvastatin". Studies were included without any restrictions on language, sex, age or publication date. These studies and relevant references cited therein were reviewed. Both abstracts and full manuscripts were considered. Moreover, no patient and public involvement should be ethically stated in this meta-analysis.

\section{Inclusion and exclusion criteria}

Studies were eligible for inclusion as the following criteria: (I) they were RCTs; (II) they evaluated the clinical efficiency of statin therapy in patients with $\mathrm{PH}$; (III) the trials provided data on at least one outcome of interest: PAP, exercise tolerance (6MWD), cardiac index (CDI), low-density lipoprotein (LDL), all-cause mortality and adverse events; (IV) if the same patient group appeared in other publications, only the latest or complete report was incorporated.

\section{Data extraction and management}

Two independent reviewers (F Chen and M Yang) separately screened the titles and abstracts, performed duplicate checking, and reviewed full articles that met the inclusion criteria. Data were independently abstracted from each identified reference with a predesigned review form. Disagreement was resolved by consensus with the third author (C Wan). The details from each study included general characteristics of the study (publication year, area, study design), participants (age, gender, number of patients in treatment and control group, diagnosis, PAP and LDL before treatment) and intervention (statin types, dose, background treatment and duration). We extracted data on the following outcomes: PAP, exercise tolerance (6MWD), 
CDI, LDL, adverse events and all-cause mortality.

\section{Quality assessment}

We assessed the risk of bias of each fully published trial according to the Cochrane risk of bias tool. The main domains were checked, including random sequence generation (selection bias), allocation concealment (selection bias), blinding of participants and personnel (performance bias), blinding of outcome assessment (detection bias), incomplete outcome data (attrition bias), selective reporting (reporting bias), and other biases. The judgments were expressed as "low risk", "high risk", or "unclear risk" of bias. Any disagreements were resolved by discussion and consensus.

\section{Statistical analysis}

The data analysis was performed with Stata 12.0 (StataCorp, College Station, TX, USA). We used the randomeffect model to conduct the meta-analysis and assessed heterogeneity with the $\mathrm{I}^{2}$ statistic and the fixed-effect model was used when the value of $\mathrm{P}>0.1$. The weighted mean difference (WMD) and $95 \%$ confidence intervals (CIs) were calculated with continuous data. When the different measurements became variable, standardized mean difference (SMD) was used. We performed subgroup analysis to estimate the clinical efficacy of statins in trials enrolling patients with concomitant COPD and patients diagnosed with PAH. When assessment of the influences of individual studies on the pooled effects was necessary, sensitivity analysis was conducted by withdrawing trials one by one. $\mathrm{P}$ value less than 0.05 was considered statistical significance.

\section{Results}

\section{Search results}

Our literature search found 783 potential relevant publications and 391 were removed because of duplication. Of the remains, 16 clinic trials comparing the treatment effects of statins with placebo in $\mathrm{PH}$ patients were identified. Ultimately, nine RCTs completely fulfilled the criteria for consideration and the data in which were extracted to further analysis (17-25). The search process is shown in Figure 1. All the selected studies were shown in English and published during 2008-2017. And publishing areas were as follows: Brazil, Taiwan, Germany, United States, China, Iran and India. The clinical features of included studies are shown in Table 1.

\section{Patients}

A total of $657 \mathrm{PH}$ patients were recruited to these 9 trials; 330 were randomized to the statin group and 327 to the control group. Mean age ranged from 33 to 72 years. Study duration ranged from 12 weeks to 6 months. The subjects were permitted to receive basic treatment such as diuretics, long-acting muscarinic antagonists, long-acting $\beta 2$-agonists, oxygen supplement and anticoagulant therapy. Four types of statins were investigated: atorvastatin, pravastatin, rosuvastatin and simvastatin.

\section{Trial quality}

The quality of the included studies was generally high. Methods of randomization and allocation concealment were adequately addressed in the majority of trials. Among the 9 included studies, 7 were double-blind, 1 was triple-blind and the remaining 1 was unclear. The blinding of participants and outcome assessors was reported in all studies, reflecting an overall low risk of detection bias. A summary of the "Risk of bias" assessment is presented in Figure 2.

\section{Clinic outcomes}

\section{PAP}

Data on PAP were available in five studies (18,21-23,25). Overall there was a significant decrease of PAP in $\mathrm{PH}$ patients treated with statins compared with placebo group (WMD $=-3.51,95 \% \mathrm{CI}:-5.35$ to $-1.68 ; \mathrm{P}<0.001$ ) (Figure 3). There was a small heterogeneity among studies $\left(\mathrm{I}^{2}=5.5 \%\right)$. In the subgroup analysis, there was a significant improvement of PAP in COPD patients (WMD $=-4.13$, 95\% CI: -6.27 to $-1.99 ; \mathrm{P}<0.001$ ) (Figure $4 A$ ), but not in $\mathrm{PAH}(\mathrm{WMD}=3.49,95 \% \mathrm{CI}:-2.03$ to $9.02 ; \mathrm{P}=0.215$ ) (Figure 4B).

\section{Exercise capacity}

Most of included studies reported $6 \mathrm{MWD}$, an indicator of exercise capacity. In this study, 6MWD were extracted from five trials $(19-21,23,24)$. There was no significant benefit in 6MWD with statins in $\mathrm{PH}$ subjects (WMD $=-2.66$, 95\% CI: -20.11 to $14.79 ; \mathrm{P}=0.765$ ) (Figure 3). Statistical heterogeneity was small $\left(\mathrm{I}^{2}=9.0 \%\right)$. In subgroup analysis, 


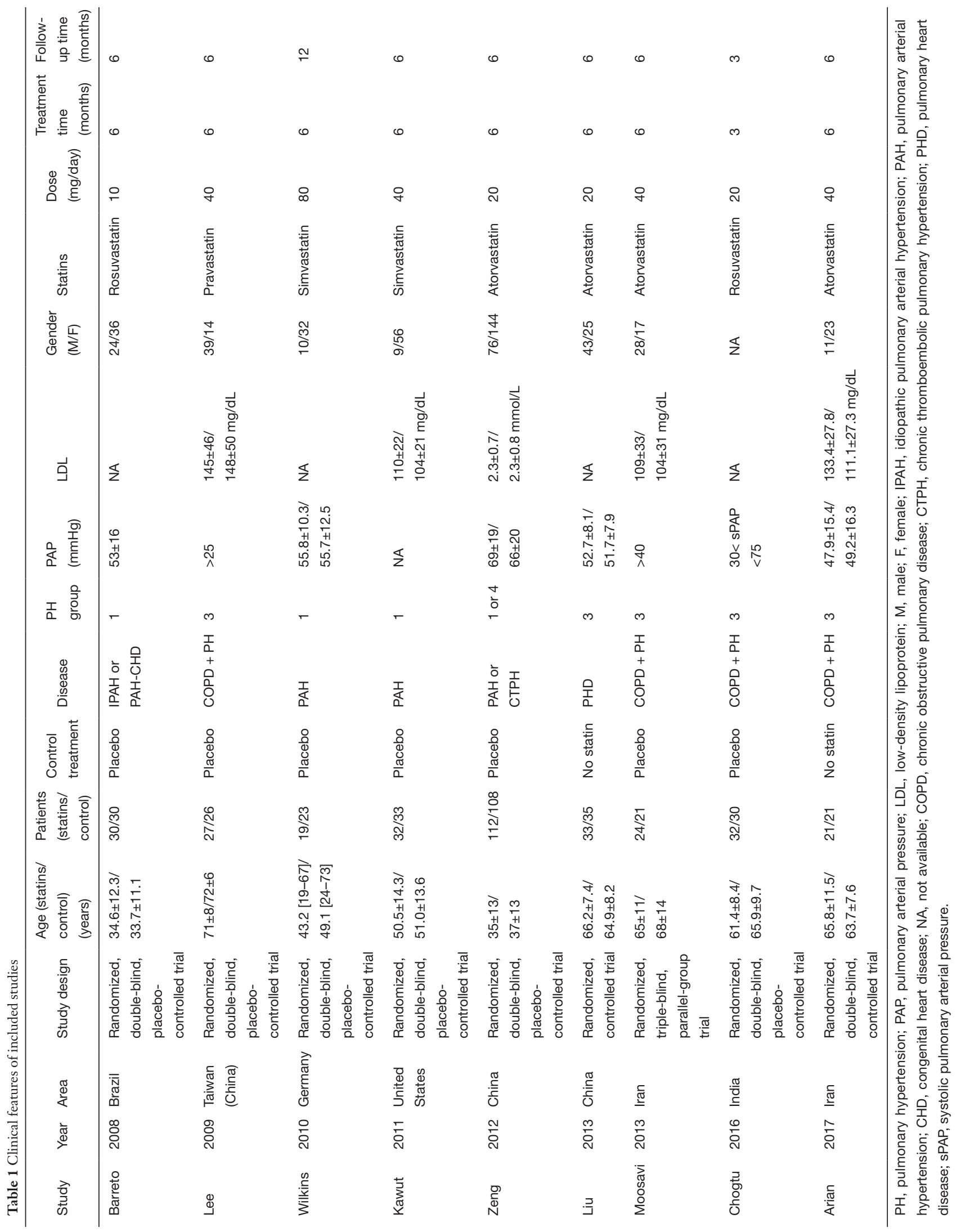




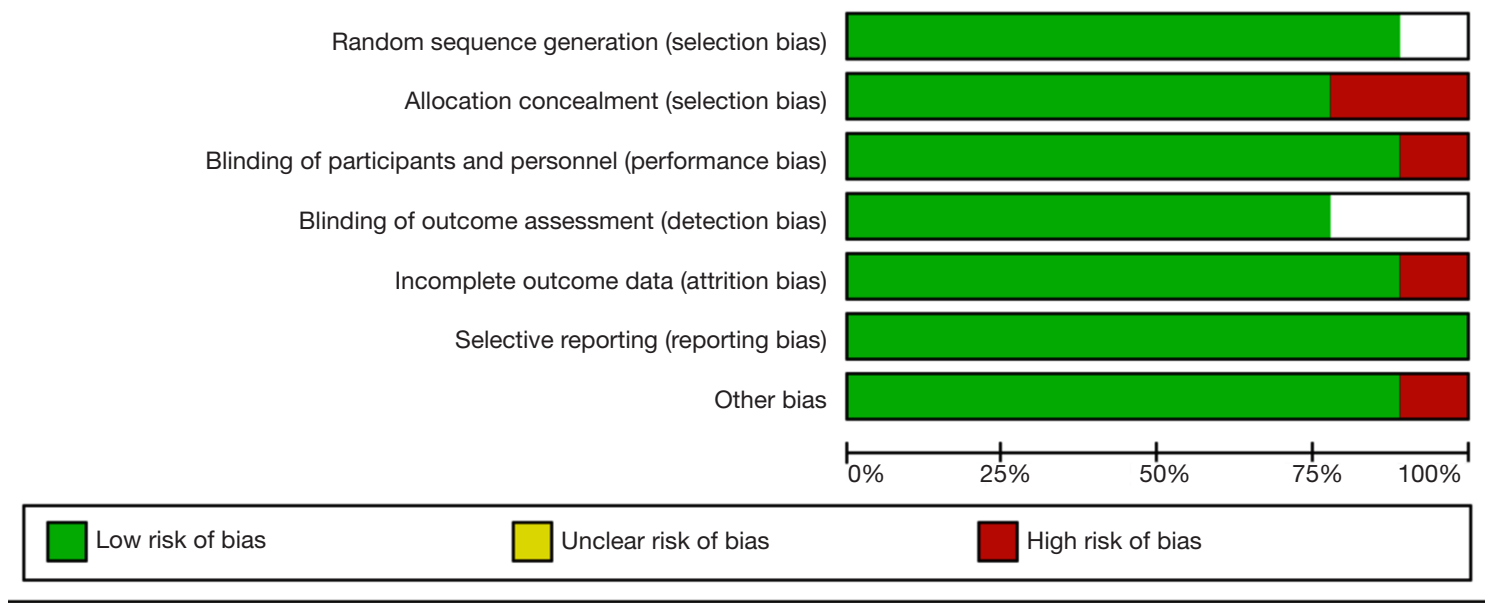

Figure 2 Risk of bias graph.

groups. There was no statistically significant difference in all-cause mortality ( $\mathrm{RR}=0.77,95 \% \mathrm{CI}: 0.36$ to 1.65 ; $\mathrm{P}=0.514$ ) (Figure 3). The heterogeneity among trials was not significant $\left(\mathrm{I}^{2}=0.0 \%\right)$.

\section{Sensitivity analysis}

The leave-one-out sensitivity analysis for the effect of statins on PAP in PH patients that was performed by using both random and fixed effect models, and practically the same outcomes were found. No pooled results or heterogeneity of this meta-analysis was significantly altered (Figure 5).

\section{Discussion}

This systematic review and meta-analysis assessed the efficacy and safety of statins in PH with nine RCTs, which yielded the following outcomes. First, statin therapy could significantly reduce PAP, as well as LDL level in patients with $\mathrm{PH}$, whereas $6 \mathrm{MWD}$ and CDI showed no statistical amelioration. Second, there were nonsignificant trends toward rise of the incidence rate of adverse events and allcause mortality in a short term. Third, according to the subgroup analysis, there was an obvious reduction of PAP in $\mathrm{PH}$ due to COPD, but not PAH, treated with statins.

$\mathrm{PH}$, means an abnormal augment of PAP due to elevated pulmonary vascular resistance, whose main vascular changes are vasoconstriction, thrombosis and vascular remodeling associated with smooth-muscle cell and endothelial-cell proliferation (4). Since the pleiotropic properties with regard to cardiovascular system of statin drugs, a couple of researches have paid attentions to statin therapy in $\mathrm{PH}$. The outcomes concerning PAP in this study were in agreement with that of several studies applying animal models (26-28). Carlin et al. revealed that some sorts of statins such as Fluvastatin could reduce PAP in PH rats (26). The finding of Li et al. showed that rosuvastatin administration could ameliorate mean PAP in Monocrotaline-induced $\mathrm{PAH}$ in rats, which might result from the regulation of Rho-associated coiled-coil-containing kinase 1 (ROCK-1), proliferating cell nuclear antigen (PCNA), and endothelial nitric oxide synthase (eNOS) expression (27). That is to say, the present outcomes confirm and extend the previous outcomes regarding the favorable impacts of statins on PAP. Also, the potential mechanisms deserve attention, in the included RCTs of this meta-analysis, Lee et al. inferred that statins inhibiting the generation of endothelin 1 (ET-1) was one of the possible reasons, as urinary ET-1 levels were significantly reduced in pravastatin-treated patients (18). Combined with analyses of antecedent researches, it is reasonable to infer that statins decrease PAP by prompting apoptosis, an important physiological process to reverse pulmonary artery remodeling, inhibiting smooth-muscle cell proliferation, promoting secretion of vasodilators such as prostacyclin and nitric oxide, inhibiting endothelin generation and anti-inflammatory, etc. (29-32).

However, not all the related researches favor statins' improving PAP, making us consider possible reasons. In the RCT conducted by Moosavi et al., Atorvastatin therapy did not notably ameliorate the systolic PAH (SPAH) in $\mathrm{PH}$ and they got an idea that, small sample size could be 


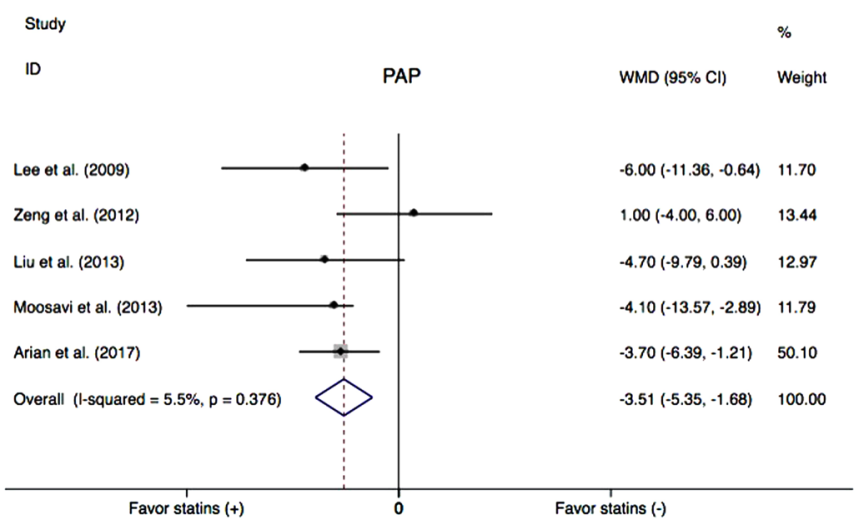

Study

ID

CDI

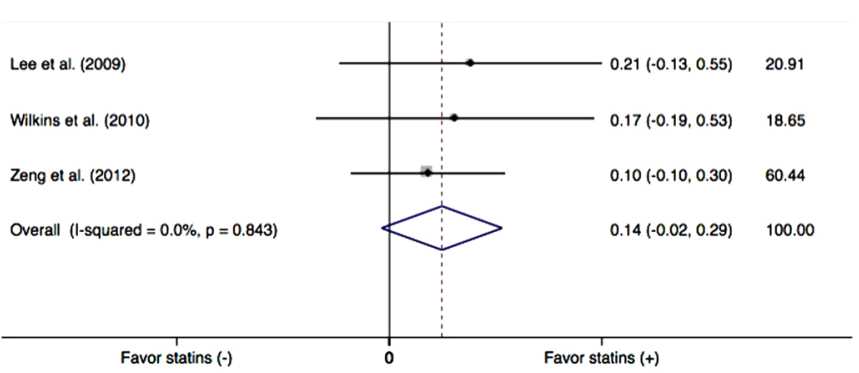

Study

ID

Barreto et al. (2008)

Wilkins et al. (2010)

Kawut et al. (2011)

Zeng et al. (2012)

Chogtu et al. (2016)

Lee et al. (2009)

Moosavi et al. (2013)

Overall (I-squared $=0.0 \%, p=0.787$ )

Favor statins (+)

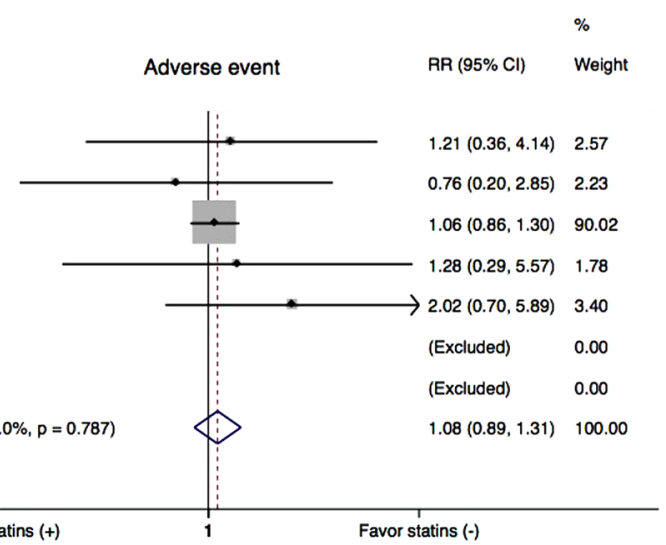

Study
ID

6MWD

WMD $(95 \% \mathrm{CI})$

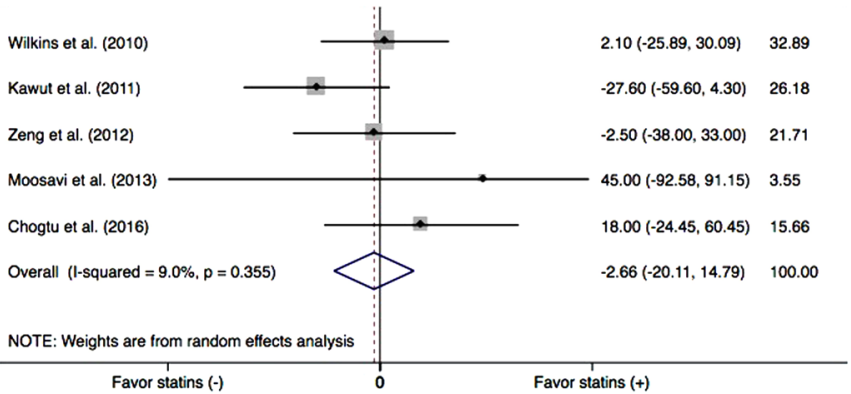

Study

ID

LDL

$\operatorname{SMD}(95 \% \mathrm{Cl}) \quad$ Weight

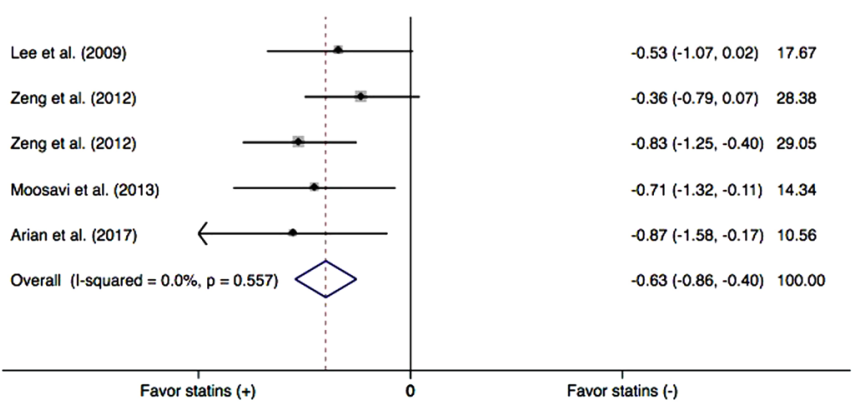

Study

ID

All-cause mortality

RR $(95 \%$ Cl) Weight

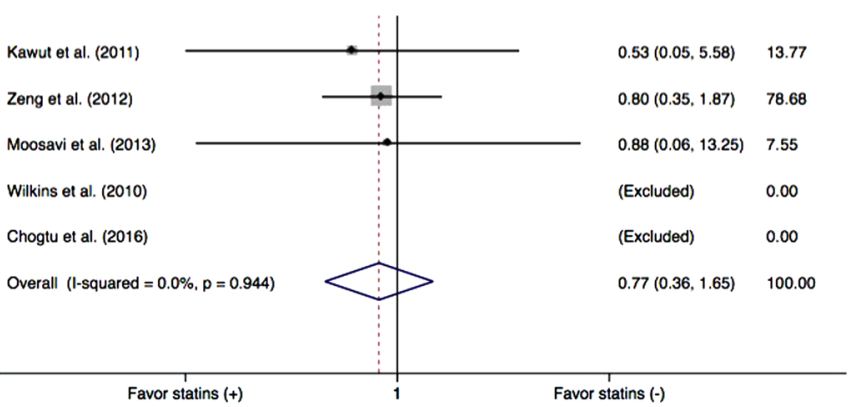

Figure 3 Summary effects of statins in PH. PAP, pulmonary arterial pressure; WMD, weighted mean difference; CI, confidence interval; 6MWD, 6-minute walking distance; CDI, cardiac index; LDL, low-density lipoprotein; SMD, standardized mean difference; RR, risk ratio; $\mathrm{PH}$, pulmonary hypertension. 
A

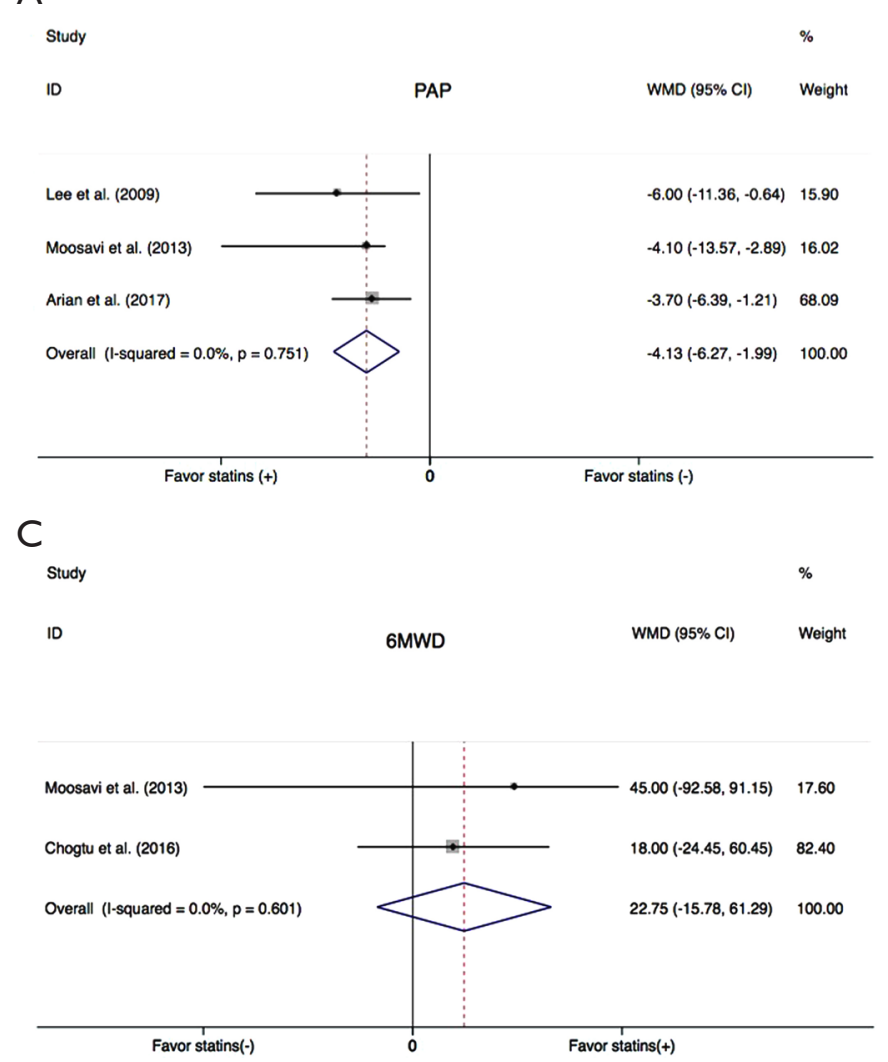

B

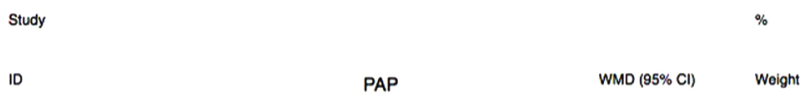

D

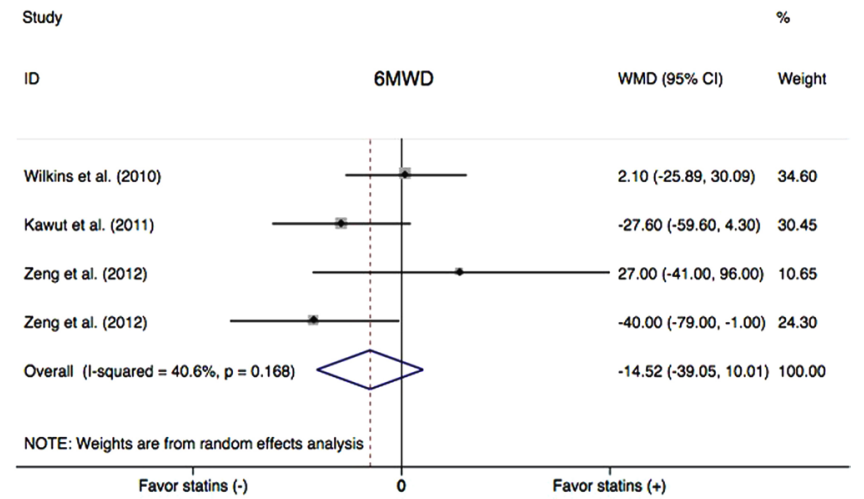

Figure 4 Summary effects of statins on PAP (A,B) and 6-minute walk distance (C,D) in COPD (A,C) and PAH (B,D). PAP, pulmonary arterial pressure; WMD, weighted mean difference; $\mathrm{CI}$, confidence interval; $6 \mathrm{MWD}$, 6-minute walking distance; $\mathrm{PAH}$, pulmonary arterial hypertension; COPD, chronic obstructive pulmonary disease.

a main account for it (23). Given the discordant results, we can make a speculation that results might be affected by the sorts and dosages of statins, clinic types of $\mathrm{PH}$, background diseases, other drugs, duration and sample size, etc. For instance, previous study showed that pravastatin, instead of simvastatin, slowed down the progression of $\mathrm{PH}$ (33). Moreover, Carlin et al. reported that some sorts of statins like fluvastatin may be more effective in PAP (26). Statins differ in metabolism, pharmacological lipophilicity, structure and solubility, which may give rise to different efficacy in PH. In the five included RCTs concerning PAP, four applying atorvastatin showed improvements in PAP except Zeng et al., who used atorvastatin at $10 \mathrm{mg} /$ day whereas others used at 20 or $40 \mathrm{mg}$ per day. This might indicate that atorvastatin plays a protective part in $\mathrm{PH}$, and the dosage of significant effects should not be lower than $20 \mathrm{mg}(18,21-23)$. In addition, there would be biases because of PH patients' older ages and background diseases. This study excluded background diseases, since severe gastrointestinal disorders and liver dysfunction could undermine the absorption and metabolism of drugs. Although it was suggested that patients with COPD at elevated cardiovascular risk might be the appropriate subgroup for statins $(34,35)$, potential impacts of cardiovascular diseases remain controversial. Of course, drug interaction cannot be neglected, such as the interplay between statins and clopidogrel, being used in cardiovascular diseases frequently, consequently promoting platelet aggregation, which does harm to cardiovascular system, as well as weakens beneficial effects of statins. Potential influencing factors above could also explain the unexpected outcomes of $6 \mathrm{MWD}$, which correlates with markers of disease severity in $\mathrm{PH}$, such as pulmonary haemodynamics (36). According to the pathogenesis, the improvement of $6 \mathrm{MWD}$ may be later than that of PAP, indicating a longer duration is needed for the change. And another plausible explanation for it was that $6 \mathrm{MWD}$ depends largely on motivation and encouragement, making 


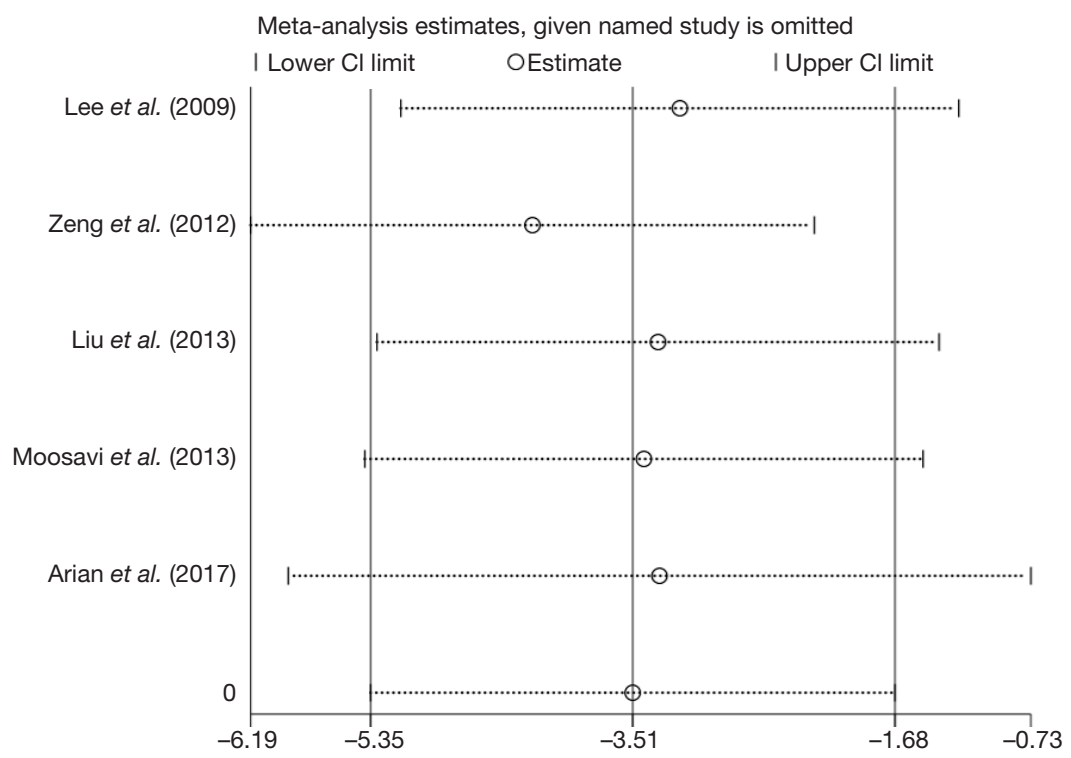

Figure 5 The leave-one-out sensitivity analysis for the effect of statins on PAP in PH patients. CI, confidence interval; PAP, pulmonary arterial pressure; $\mathrm{PH}$, pulmonary hypertension.

it difficult to standardize (37). On basis of information above, a well-conducted study is necessary.

$\mathrm{PAH}$ and $\mathrm{PH}$ due to COPD, as two clinic subtypes of $\mathrm{PH}$, differ in primary causes and pathogenesis. Previous studies have indicated the effects of statin therapy in PAH and COPD were inconsistent $(10,17,24,38)$. In our metaanalysis, $\mathrm{PH}$ due to COPD patients appeared to benefit more than the $\mathrm{PAH}$ from statins. There were possible reasons. First, ever-increasing researches have proved that COPD is a chronic systemic inflammation $(35,39)$. Inflammation and hypoxia impair pulmonary blood vessels, ultimately leading to PH. It is noticeable, COPD patients conspicuous changes in pulmonary vascular remodeling (40). Moreover, anti-inflammation and maintaining endothelial cell function properties make statins favourable to $\mathrm{PH}$ due to COPD $(5,7)$. Actually, statin therapy has played a major role in the prevention and reversal of PH secondary to hypoxia in studies. Second, PAH as the group 1 of $\mathrm{PH}$, which comprises various causes of diseases, such as idiopathic (IPAH), heritable forms, congenital systemicto-pulmonary shunts and other conditions (20). It is worth nothing that, the nine included RCTs, only one (Zeng et al.) reported the change value of PAP in whole $\mathrm{PH}$ patients and the subgroups of PAP in PAH patients (connective tissue disorder and congenital heart disease), combined with the research of Rysz-Górzynska et al. showing that statins treatment had nonsignificant change of PAP among PAH patients (11). It is possible that these mixed diseases included in PAH group limit the overall efficacy of statins. However, the direct pulmonary vascular effect of statin in PAH is unclear. Therefore, it is advisable to explore the causes of PAH simultaneously. On basis of the ever-growing incidence rate of COPD, it would be promising to get more efficient and valuable results if future studies recruit more $\mathrm{PH}$ combined with COPD patients to determine the role of statin therapy.

In terms of safety, common statin-induced adverse effects containing liver impairing, bleeding, irregular menstrual cycles, abdominal distention and their likes (10). Several rare but serious, such as rhabdomyolysis, new-onset diabetes, and possibly acute kidney injury make it necessary to determine the safety of statin therapy in $\mathrm{PH}$ concerning does, type and time (41). Nakano et al. suggested statin therapy was relatively safe among $\mathrm{PH}$ patients, which was similar to this study's (42). Further, there was a research revealed statins' significant chemopreventive effect to reduce cancer risk in COPD $(42,43)$. However, in view of the short-term duration our finding, while $\mathrm{PH}$ requiring long-term drug therapy, we should consider the results dialectically. Liang et al. made a subgroup analysis showing that fluvastatin had a significant impact on liver, and the incidence rate of adverse events increased when the dose of statins more than $40 \mathrm{mg}$. They also indicated side effects associated with statin therapy were more obvious in the first 
2 years (44). It is worth nothing that this study only assessed the liver function, with the recruited patients were not all suffering PH. Nonetheless, future researches can still base on it, further optimize the inclusion and exclusion criteria, increase indicators to better assess the safety of statins, especially long-term safety.

\section{Limitation}

This systematic review and meta-analysis has limitations. First, the overall scale of the contributing studies was small and the confidence intervals were wide, which are limited by the source data. Second, several important specific outcomes of COPD and PAH including adverse events and all-cause mortality could not be analyzed in subgroups, due to lack of reported outcomes in trials. Third, despite generally similar demographic characteristics and minimal evidence of heterogeneity among these RCTs, some confounding factors such as different types and doses of statins, which might have caused potential biases. Fourth, our study only includes three subtypes of $\mathrm{PH}$ patients (types 1, 3 and 4), therefore the outcomes may not suitable for all groups of the PH patients. Future clinic trials with rigorously predefined patient inclusion criteria are warranted to better research the potential role of statin therapy in $\mathrm{PH}$.

\section{Conclusions}

This systematic review and meta-analysis showed that with routine treatment, adding statins could be beneficial for PAP, but not 6MWD and CDI in PH. And during short-term therapy, adverse events and mortality were not apparent, suggesting a relative safety. It is advisable that more future researches should focus on the efficacy and safety of statin therapy in different subtypes of $\mathrm{PH}$.

\section{Acknowledgments}

Funding: This study was supported in part by grant $18 \mathrm{PJ} 410$ from the Health and Family Planning Commission of Sichuan Province and grant 2014SZ0220 from the Science and Technology Support Program of Sichuan Province and grant 2016YFC0901100 from the National Key Research and Development Program of China.

\section{Footnote}

Conflicts of Interest: The authors declare they have no conflict of interests.

Ethical Statement: The authors are accountable for all aspects of the work in ensuring that questions related to the accuracy or integrity of any part of the work are appropriately investigated and resolved.

\section{References}

1. "2015 ESC/ERS Guidelines for the diagnosis and treatment of pulmonary hypertension. The Joint Task Force for the Diagnosis and Treatment of Pulmonary Hypertension of the European Society of Cardiology (ESC) and the European Respiratory Society (ERS)." Nazzareno Galie, Marc Humbert, Jean-Luc Vachiery, Simon Gibbs, Irene Lang, Adam Torbicki, Gerald Simonneau, Andrew Peacock, Anton Vonk Noordegraaf, Maurice Beghetti, Ardeschir Ghofrani, Miguel Angel Gomez Sanchez, Georg Hansmann, Walter Klepetko, Patrizio Lancellotti, Marco Matucci, Theresa McDonagh, Luc A. Pierard, Pedro T. Trindade, Maurizio Zompatori and Marius Hoeper. Eur Respir J 2015; 46: 903-975. Eur Respir J 2015;46:1855-6.

2. Kovacs G, Dumitrescu D, Barner A, et al. Definition, clinical classification and initial diagnosis of pulmonary hypertension: Updated recommendations from the Cologne Consensus Conference 2018. Int J Cardiol 2018;272S:11-9.

3. Sharma M, Pinnamaneni S, Aronow WS, et al. Existing drugs and agents under investigation for pulmonary arterial hypertension. Cardiol Rev 2014;22:297-305.

4. Hoeper MM, Ghofrani HA, Grunig E, et al. Pulmonary Hypertension. Dtsch Arztebl Int 2017;114:73-84.

5. Bradbury P, Traini D, Ammit AJ, et al. Repurposing of statins via inhalation to treat lung inflammatory conditions. Adv Drug Deliv Rev 2018;133:93-106.

6. Wilson SH, Herrmann J, Lerman LO, et al. Simvastatin preserves the structure of coronary adventitial vasa vasorum in experimental hypercholesterolemia independent of lipid lowering. Circulation 2002;105:415-8.

7. Kwak B, Mulhaupt F, Myit S, et al. Statins as a newly recognized type of immunomodulator. Nat Med 2000;6:1399-402.

8. Ren J, Liu W, Li GC, et al. Atorvastatin Attenuates Myocardial Hypertrophy Induced by Chronic Intermittent Hypoxia In Vitro Partly through miR-31/PKC epsilon Pathway. Curr Med Sci 2018;38:405-12. 
9. Kao PN. Simvastatin treatment of pulmonary hypertension: an observational case series. Chest 2005; 127:1446-52.

10. Anand V, Garg S, Duval S, et al. A systematic review and meta-analysis of trials using statins in pulmonary arterial hypertension. Pulm Circ 2016;6:295-301.

11. Rysz-Górzynska M, Gluba-Brzózka A, Sahebkar A, et al. Efficacy of Statin Therapy in Pulmonary Arterial Hypertension: A Systematic Review and Meta-Analysis. Sci Rep 2016;6:30060.

12. Wang L, Qu M, Chen Y, et al. Statins Have No Additional Benefit for Pulmonary Hypertension: A MetaAnalysis of Randomized Controlled Trials. PLoS One 2016;11:e0168101.

13. Zhang Y, Zeng W, Cheng S, et al. Efficacy and Safety of Statins for Pulmonary Hypertension: A Meta-Analysis of Randomised Controlled Trials. Heart Lung Circ 2017;26:425-32.

14. Holzhauser L, Hovnanians N, Eshtehardi P, et al. Statin therapy improves survival in patients with severe pulmonary hypertension: a propensity score matching study. Heart Vessels 2017;32:969-76.

15. King WT, Day RW. Treatment of pediatric pulmonary hypertension with simvastatin: an observational study. Pediatr Pulmonol 2011;46:261-5.

16. Wang P, Yang J, Yang Y, et al. Effect of azithromycin in combination with simvastatin in the treatment of chronic obstructive pulmonary disease complicated by pulmonary arterial hypertension. Pak J Med Sci 2017;33:260-4.

17. Barreto AC, Maeda NY, Soares RP, et al. Rosuvastatin and vascular dysfunction markers in pulmonary arterial hypertension: a placebo-controlled study. Braz J Med Biol Res 2008;41:657-63.

18. Lee TM, Chen CC, Shen HN, et al. Effects of pravastatin on functional capacity in patients with chronic obstructive pulmonary disease and pulmonary hypertension. Clin Sci (Lond) 2009;116:497-505.

19. Wilkins MR, Ali O, Bradlow W, et al. Simvastatin as a treatment for pulmonary hypertension trial. Am J Respir Crit Care Med 2010;181:1106-13.

20. Kawut SM, Bagiella E, Lederer DJ, et al. Randomized clinical trial of aspirin and simvastatin for pulmonary arterial hypertension: ASA-STAT. Circulation 2011;123:2985-93.

21. Zeng WJ, Xiong CM, Zhao L, et al. Atorvastatin in pulmonary arterial hypertension (APATH) study. Eur Respir J 2012;40:67-74.

22. Liu HF, Qi XW, Ma LL, et al. Atorvastatin improves endothelial progenitor cell function and reduces pulmonary hypertension in patients with chronic pulmonary heart disease. Exp Clin Cardiol 2013;18:e40-3.

23. Moosavi SA, Raji H, Faghankhani M, et al. Evaluation of the Effects of Atorvastatin on the Treatment of Secondary Pulmonary Hypertension due to Chronic Obstructive Pulmonary Diseases: A Randomized Controlled Trial. Iran Red Crescent Med J 2013;15:649-54.

24. Chogtu B, Kuriachan S, Magazine R, et al. A prospective, randomized study: Evaluation of the effect of rosuvastatin in patients with chronic obstructive pulmonary disease and pulmonary hypertension. Indian J Pharmacol 2016;48:503-8.

25. Arian A, Moghadam SG, Kazemi T, et al. The Effects of Statins on Pulmonary Artery Pressure in Patients with Chronic Obstructive Pulmonary Disease: A Randomized Controlled Trial. J Res Pharm Pract 2017;6:27-30.

26. Carlin CM, Celnik DF, Pak O, et al. Low-dose fluvastatin reverses the hypoxic pulmonary adventitial fibroblast phenotype in experimental pulmonary hypertension. Am J Respir Cell Mol Biol 2012;47:140-8.

27. Li XL, Guan RJ, Li JJ. Attenuation of monocrotalineinduced pulmonary arterial hypertension in rats by rosuvastatin. J Cardiovasc Pharmacol 2012;60:219-26.

28. Satoh M, Satoh A. 3-Hydroxy-3-methylglutaryl (HMG)COA reductase inhibitors and phosphodiesterase type $\mathrm{V}$ inhibitors attenuate right ventricular pressure and remodeling in a rat model of pulmonary hypertension. J Pharm Pharm Sci 2009;11:118s-30s.

29. Gurbanov E, Shiliang X. The key role of apoptosis in the pathogenesis and treatment of pulmonary hypertension. Eur J Cardiothorac Surg 2006;30:499-507.

30. Taraseviciene-Stewart L, Scerbavicius R, Choe KH, et al. Simvastatin causes endothelial cell apoptosis and attenuates severe pulmonary hypertension. Am J Physiol Lung Cell Mol Physiol 2006;291:L668-76.

31. Guerard P, Rakotoniaina Z, Goirand F, et al. The HMG-CoA reductase inhibitor, pravastatin, prevents the development of monocrotaline-induced pulmonary hypertension in the rat through reduction of endothelial cell apoptosis and overexpression of eNOS. Naunyn Schmiedebergs Arch Pharmacol 2006;373:401-14.

32. Ali OF, Growcott EJ, Butrous GS, Wharton J. Pleiotropic effects of statins in distal human pulmonary artery smooth muscle cells. Respir Res 2011;12:137.

33. Rakotoniaina Z, Guerard P, Lirussi F, et al. The protective effect of HMG-CoA reductase inhibitors against monocrotaline-induced pulmonary hypertension in the 
rat might not be a class effect: comparison of pravastatin and atorvastatin. Naunyn Schmiedebergs Arch Pharmacol 2006;374:195-206.

34. Young RP, Hopkins RJ, Agusti A. Statins as adjunct therapy in COPD: how do we cope after STATCOPE? Thorax 2014;69:891-4.

35. Zhang W, Zhang Y, Li CW, et al. Effect of Statins on COPD A Meta-Analysis of Randomized Controlled Trials. Chest 2017;152:1159-68.

36. Lee WT, Peacock AJ, Johnson MK. The role of per cent predicted 6-min walk distance in pulmonary arterial hypertension. Eur Respir J 2010;36:1294-301.

37. Frost AE, Langleben D, Oudiz R, et al. The 6-min walk test $(6 \mathrm{MW})$ as an efficacy endpoint in pulmonary arterial hypertension clinical trials: demonstration of a ceiling effect. Vascul Pharmacol 2005;43:36-9.

38. Reed RM, Iacono A, DeFilippis A, et al. Statin therapy is associated with decreased pulmonary vascular pressures in severe COPD. COPD 2011;8:96-102.

39. Janda S, Park K, FitzGerald JM, et al. Statins in COPD: a systematic review. Chest 2009;136:734-43.

Cite this article as: Chen F, Yang M, Wan C, Liu L, Chen L. Efficacy and safety of statin therapy in pulmonary hypertension: a systematic review and meta-analysis. Ann Transl Med 2019;7(23):786. doi: 10.21037/atm.2019.11.19
40. Barberà JA. Mechanisms of development of chronic obstructive pulmonary disease-associated pulmonary hypertension. Pulm Circ 2013;3:160-4.

41. Li DQ, Kim RB, McArthur E, et al. Statin Safety in Chinese: A Population-Based Study of Older Adults. PLoS One 2016;11:e0150990.

42. Nakano K, Matoba T, Koga JI, et al. Safety, Tolerability, and Pharmacokinetics of NK-104-NP A Multicenter, Randomized, Placebo-Controlled Phase I InvestigatorInitiated Trial for Intravenous Administration of Pitavastatin-Loaded PLGA Nanoparticles (NK-104NP) in Healthy Japanese Male Subjects. Int Heart J 2018;59:1015-25.

43. Chen CC, Hsu YP, Liu JC, et al. Statins DoseDependently Exert Significant Chemopreventive Effects Against Various Cancers in Chronic Obstructive Pulmonary Disease Patients: A Population-Based Cohort Study. J Cancer 2016;7:1892-900.

44. Liang X, He Q, Zhao Q. Effect of Stains on LDL Reduction and Liver Safety: A Systematic Review and Meta-Analysis. Biomed Res Int 2018;2018:7092414. 